\title{
BIOLOGICAL CONTROL
}

\section{Distribuição de Ovos de Plutella xylostella (L.) (Lepidoptera: Plutellidae) e o Parasitismo por Trichogramma pretiosum Riley (Hymenoptera: Trichogrammatidae)}

\author{
Hugo B Zago ${ }^{1}$, Reginaldo Barros ${ }^{1}$, Jorge B Torres ${ }^{1}$, Dirceu Pratissoli ${ }^{2}$ \\ ${ }^{1}$ Unidade Acadêmica de Serra Talhada, Univ Federal Rural de Pernambuco, Fazenda Saco s/n, Zona Rural, CP 63, \\ 56900-000,Serra Talhada,PE, Brasil; rbarros@depa.ufrpe.br; torres@depa.ufrpe.br \\ ${ }^{2}$ Centro de Ciências Agrárias, Univ Federal do Espírito Santo, Alto Universitário s/n, CP 16, 29500-000 Alegre, ES, \\ Brasil; pratissoli@cca.ufes.br
}

Edited by Dori Nava - EMBRAPA

Neotropical Entomology 39(2):241-247 (2010)

Egg Distribution of Plutella xylostella (L.) (Lepidoptera: Plutellidae) and the Parasitism by Trichogramma pretiosum Riley (Hymenoptera: Trichogrammatidae)

\begin{abstract}
Oviposition site preference of Plutella xylostella (L.) and the parasitism by Trichogramma pretiosum Riley on cabbage plants at pre- and post-head formation were investigated in commercial cabbage field. Groups of six plants were randomly caged. In each cage three pairs of $24 \mathrm{~h}$-old $P$. xylostella moths were released, followed by 1,200 T. pretiosum females released $12 \mathrm{~h}$ later. After $48 \mathrm{~h}$ from parasitoid release, plants were harvested and fully inspected with the oviposition mapped according to the leaf position and their parts (basal, median and border) and upper and bellow leaf surfaces. Moth oviposition and egg parasitism were equally distributed across the plant leaves at the pre-head formation stage. At this stage, the basal part and the upper leaf surface were the preferred places for oviposition and egg parasitism, respectively. Cabbage plants at the post-head formation stage exhibited greater oviposition and egg parasitism in the inner leaf attached to the head. At this stage, the leaf border and the upper leaf surface were the preferred places for oviposition and egg parasitism, respectively. The infestation of $P$. xylostella on cabbage can be determined by egg counting at the pre- and post-head formation stages at the basal part of the leaves or at the border of the leaf attached to the head, respectively. And, in both plant stages parasitized eggs by T. pretiosum are more likely to be found at the upper leaf surface.
\end{abstract}

KEY WORDS: Diamondback moth, biological control, egg parasitoid, field parasitism

O cultivo de brássicas no Brasil e, em especial no Agreste de Pernambuco, é usualmente, realizado por pequenos produtores em plantios escalonados no tempo visando atender a demanda de mercado. O cultivo geralmente é simultâneo com diversas variedades de brássicas, formando um mosaico com disponibilidade abundante de hospedeiros para pragas. Esse sistema de cultivo, com múltiplos hospedeiros disponíveis em todas as épocas do ano, favorece a ocorrência de pragas como a traça-das-crucíferas, Plutella xylostella (L.), que é pragachave das brássicas em todo o mundo (Godin \& Boivin 1998). A disponibilidade de hospedeiros, aliada à grande capacidade migratória da praga, alto índice de fecundidade e ciclo curto, tem acarretado perdas na produção apesar do uso intenso de inseticidas para o seu controle. Ainda, somado à seleção de populações resistentes a inseticidas, tem inviabilizado a produção de brássicas em países como a Coréia e Paquistão (Kwon 2004, Sayyed et al 2005).

Para viabilizar o manejo de $P$. xylostella, várias alternativas de controle têm sido investigadas (Shelton \&
Nault 2004, Altre \&, Somvanshi 2006, Charleston et al 2006, Ibrahim \& Kim 2006). Dentre elas, o controle biológico com parasitóides tem mostrado potencial de uso, pois aproximadamente 90 espécies de parasitóides de $P$. xylostella já foram identificadas, entre os quais, Trichogramma spp. destacam-se pela frequência da constatação e as altas taxas de parasitismo (Godin \& Boivin 1998).

Na América do Sul, há relatos de Trichogramma pretiosum Riley parasitando ovos de $P$. xylostella somente no Brasil (Zucchi \& Monteiro 1997). Entretanto, alguns fatores podem interferir na eficiência do parasitóide de ovos Trichogramma, como o habitat do hospedeiro e a localização do ovo hospedeiro na planta hospedeira (Vinson 1997). As características da estrutura da planta, como a quantidade de tricomas, cerosidade e forma da superfície das folhas e o tamanho da planta, podem influenciar o comportamento de busca pelo hospedeiro de Trichogramma, reduzindo a taxa de parasitismo (Romeis et al 2005, Olson \& Andow 2006). Além disso, essas características da planta podem induzir 
mudanças comportamentais da praga-alvo, como alteração na escolha do local de oviposição para escapar do ataque de inimigos naturais ou preferência por locais mais tenros, que forneçam mais nutrientes para os seus descendentes (Shiojiri \& Takabayashi 2003, Reddy et al 2004, Hamilton et al 2005, Badenes-Perez et al 2006). Esses locais podem coincidir com áreas da planta que apresentam barreiras morfológicas, como a grande intensidade de tricomas que dificulta o parasitismo, principalmente por Trichogramma, dado o seu tamanho reduzido ( $>1 \mathrm{~mm})$, além de apresentarem comportamento da fase final de localização do hospedeiro por caminhamento e vôos curtos na superfície das plantas onde os ovos do hospedeiro se encontram (Romeis et al 2005, Faria et al 2008).

O objetivo deste trabalho foi investigar o efeito de duas idades de plantas de repolho, Brassica oleracea var. capitata, no padrão de oviposição de $P$. xylostella e no parasitismo de seus ovos por $T$. pretiosum.

\section{Material e Métodos}

Criação do hospedeiro. O hospedeiro alternativo Anagasta kuehniella (Zeller) (Lepidoptera: Pyralidae) foi criado utilizando a dieta proposta por Torres et al (1995), à base de farinha de trigo integral (60\%), fubá de milho (37\%) e levedura de cerveja (3\%).

Ovos e adultos da traça-das-crucíferas, P. xylostella, utilizados nos experimentos foram provenientes de criação em folhas de couve de produção orgânica, conforme Barros \& Vendramim (1999).

Criação de T. pretiosum. Os parasitóides utilizados nos estudos foram mantidos em ovos do hospedeiro alternativo A. kuehniella. A colônia foi iniciada com a população de $T$. pretiosum coletada em cultivos de hortaliças no município de Chã-Grande, PE (8 $14^{\prime} 24^{\prime \prime} \mathrm{S}$, 35 $25^{\circ}$ '04 'W e a $450 \mathrm{~m}$ de altitude). A confirmação da espécie T. pretiosum foi realizada pela taxonomista de Trichogrammatidae Dr ${ }^{\mathrm{a}}$ Ranyse Barbosa Querino da Silva (Embrapa Roraima, Boa Vista, RR).

Para a manutenção de T. pretiosum foi adotada a técnica desenvolvida por Parra (1997) adaptada às condições do Laboratório de Biologia da UFRPE, onde foram oferecidos ovos de A. kuehniella colados em retângulos de cartolina azul, com goma arábica. Os ovos do hospedeiro foram previamente inviabilizados com lâmpada germicida. As cartelas com os ovos do hospedeiro alternativo foram inseridas em recipientes de vidro, contendo adultos de $T$. pretiosum, idade $0-6 \mathrm{~h}$, e o parasitismo foi permitido por $24 \mathrm{~h}$. As cartelas com ovos parasitados foram armazenadas em sala climatizada (25 \pm $1^{\circ} \mathrm{C}, 70-80 \% \mathrm{UR}$, fotofase de $12 \mathrm{~h}$ ).

Oviposição de Plutella e parasitismo por Trichogramma no campo. $\mathrm{O}$ experimento foi instalado em cultivo comercial de repolho, $B$. oleracea var. capitata (Midori), localizado no município de Gravatá, Agreste de Pernambuco (8¹2`34`S, $35^{\circ} 34^{`} 10^{\prime \prime} \mathrm{W}$ e $500 \mathrm{~m}$ altitude). As plantas de repolho de tamanho uniforme foram selecionadas dentro do cultivo comercial para a condução do experimento e acondicionadas em gaiolas na densidade de seis plantas por gaiola. Adultos de P. xylostella com $24 \mathrm{~h}$ de idade e no início da atividade de postura foram liberados em número de três casais por gaiola às $17 \mathrm{~h}$. Decorridas $12 \mathrm{~h}$ da liberação de $P$. xylostella (i.e., na manhã do dia seguinte), foram liberadas 1.200 fêmeas de $T$. pretiosum por gaiola (proporção de 200 fêmeas do parasitóide por planta, baseada em testes preliminares). Após 48h da liberação do parasitóide, as plantas de repolho foram colhidas e levadas ao laboratório para avaliar o local de oviposição de $P$. xylostella e confirmar o parasitismo por T. pretiosum. As folhas das plantas de repolho foram enumeradas, separadas da base para o ápice (Fig 1a) e dividas em partes denominadas basal, mediana e borda (Fig 1b), além da epiderme superior e inferior. O local da folha que apresentava ovos de $P$. xylostella foi identificado e removido com auxílio de estilete, os quais foram acondicionados em placas de Petri para confirmação do parasitismo. As temperaturas no interior das
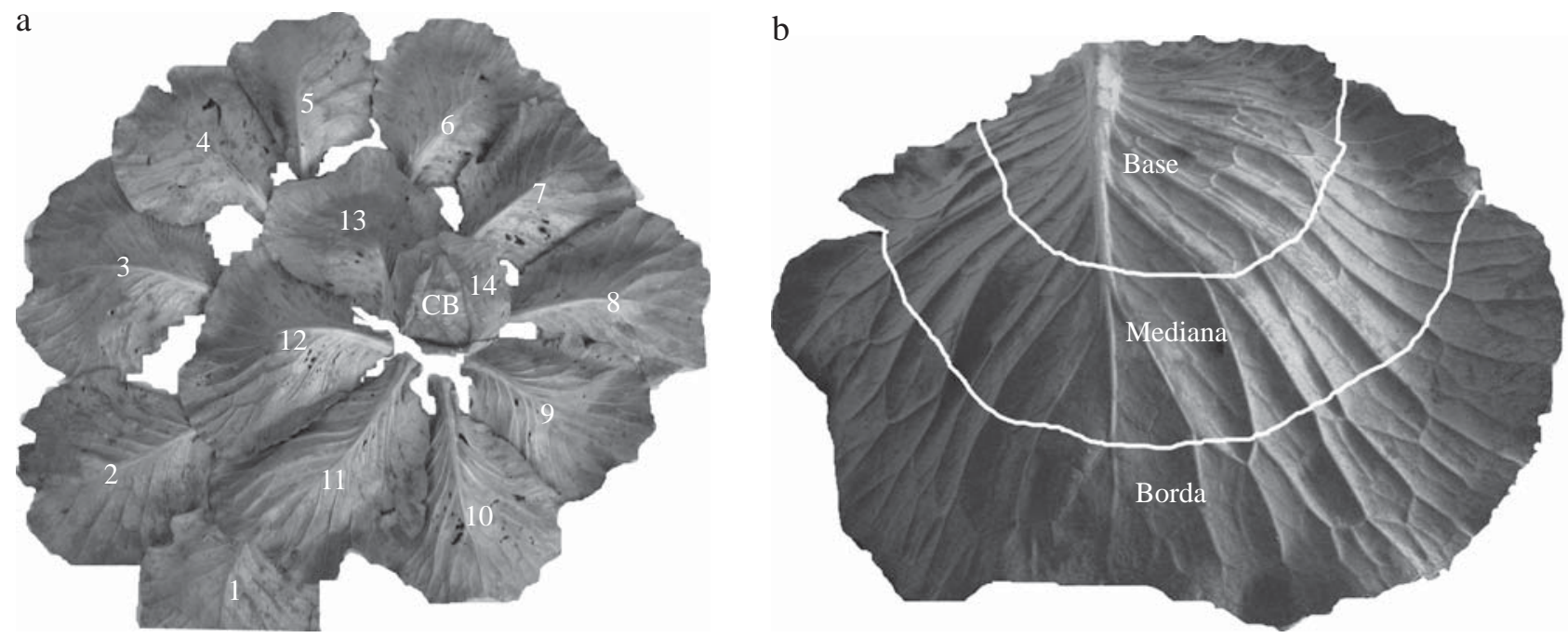

Fig 1 a) Distribuição das folhas entre a base (1) e a cabeça (CB) da planta de repolho e; b) regiões da folha de repolho, Brassica oleracea variedade capitata (Midori). 
gaiolas foram determinadas nos intervalos da realização do experimento, plantas com 30 e 60 dias, $23,7 \pm 3,28^{\circ} \mathrm{C}$ e 25,0 $\pm 4,3^{\circ} \mathrm{C}$, respectivamente, com auxílio do aparelho WatchDog datalogger (Spectrum ${ }^{\mathrm{TM}}$ Technologies, Inc., Plainfield, IL).

Os experimentos foram realizados com plantas de repolho nas idades fenológicas antes e após a formação de cabeça, correspondente a 30 e 60 dias após o transplante. Foram estabelecidas 20 e 28 repetições (gaiolas com seis plantas cada) para as idades antes e após a formação da cabeça, respectivamente, com o total de 120 e 168 plantas analisadas. Os dados referentes à distribuição da postura de P. xylostella e o número de ovos parasitados por T. pretiosum em função das partes (folhas, superfície da folha e cabeça), nas respectivas idades das plantas, foram submetidos à análise de variância (ANOVA) e as médias comparadas pelo teste de Scott-Knott com o auxílio do programa estatístico SAEG Versão 7.0 (SAEG 1997). A relação entre parasitismo e densidade de hospedeiro foi determinada via regressão entre o número de ovos parasitados e a proporção de ovos parasitados por $T$. pretiosum em função da densidade de ovos de $P$. xylostella em plantas de repolho com 30 e 60 dias após transplante, respectivamente.

\section{Resultados}

O total de 154 e 805 ovos de P. xylostella foi contabilizado para os estágios de desenvolvimento antes e após a formação da cabeça, respectivamente, e mapeados de acordo com as estruturas das plantas. O comportamento de deposição dos ovos por $P$. xylostella, bem como o parasitismo observado por T. pretiosum, foi influenciado de acordo com o estágio de desenvolvimento da planta antes e após a formação de cabeça (Figs 2, 3). As plantas de repolho com cabeça formada (i.e., 60 dias após o transplante) apresentaram maior oviposição de P. xylostella e parasitismo por T. pretiosum na folha central que envolve a cabeça, borda e epiderme superior, quando comparadas às plantas antes da formação da cabeça (i.e., 30 dias após o transplante). Plantas de repolho antes da formação da cabeça apresentavam-se com 12 folhas e haste principal definida sem indícios de formação de cabeça. Nessa idade fenológica das plantas, adultos de $P$. xylostella mostraram preferência para ovipositar na parte basal das folhas, mas não demonstraram diferenças na oviposição entre a $1^{\mathrm{a}}$ e a $12^{\mathrm{a}}$ folha, bem como entre as epidermes superior e inferior (Fig 2a). Da mesma forma comportou-se o parasitismo de ovos por T. pretiosum, apresentando taxa similar de parasitismo entre folhas e partes das folhas, com exceção do maior parasitismo observado na epiderme superior das folhas (Fig 2b).

As plantas de repolho, após formação da cabeça (i.e., 60 dias após o transplante), apresentavam-se com 1 a 12 folhas abertas e cabeça formada, pronta para a colheita. Entre as estruturas da planta, os adultos de $P$. xylostella preferiram depositar ovos na folha mais interna envolvendo a cabeça, na borda desta folha e na sua epiderme superior (Figs 3a-c). A taxa de parasitismo de ovos por T. pretiosum também se mostrou associada à oviposição de $P$. xylostella, sendo superior na folha envolvendo a cabeça (76\%), borda da folha (71\%) e epiderme superior dessa folha (60\%) (Figs 3a-c).

Em ambas as idades fenológicas das plantas houve
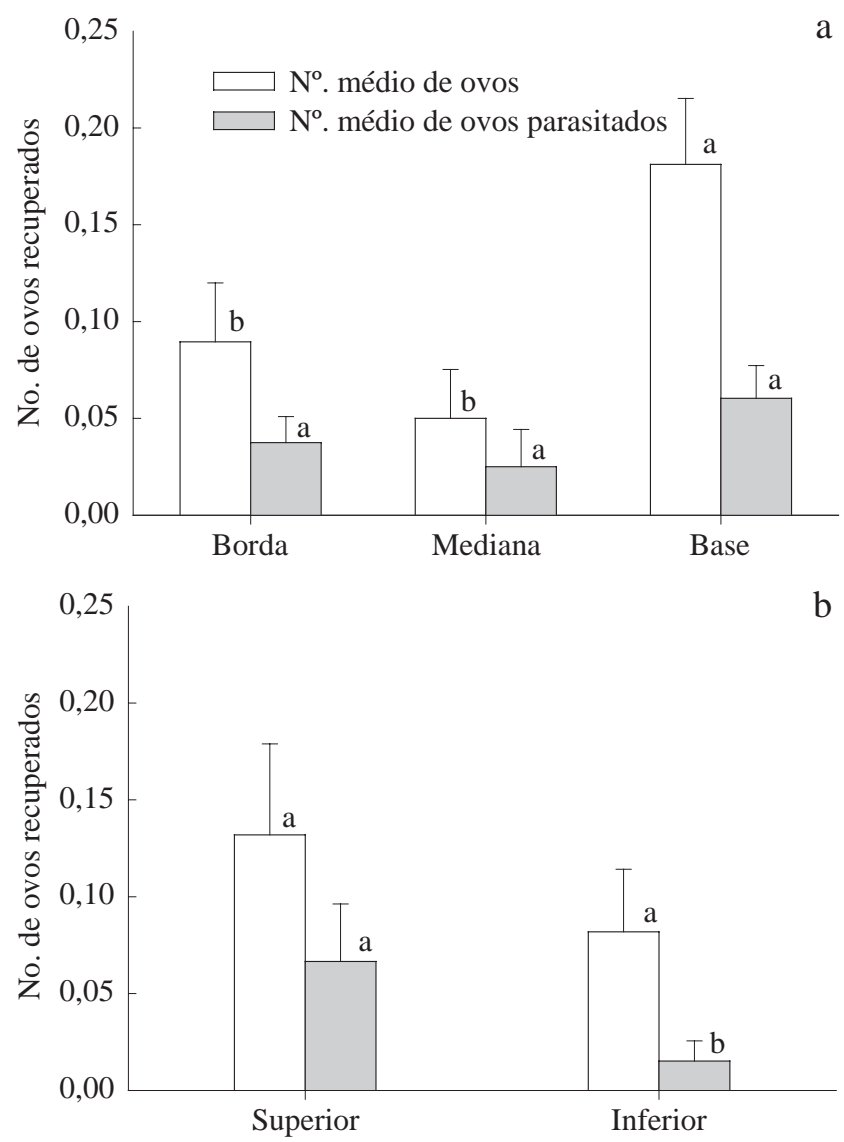

Fig 2 a) Número de ovos de Plutella xylostella e número de ovos parasitados por Trichogramma pretiosum nas respectivas partes das folhas de repolho, Brassica oleracea var. capitata (Midori) com 30 dias após o transplante; b) epiderme das folhas de plantas. As médias (+EP) seguidas de mesma letra na categoria, não diferem entre si, pelo teste de Scott-Knott a 5\% de significância.

relação significativa entre o número de ovos de $P$. xylostella parasitados por $T$. pretiosum em função do número de ovos disponíveis encontrados nas seis plantas (cada gaiola) (Figs 4a, c). Antes da formação da cabeça foram recuperados de 1 a 28 ovos por seis plantas, com 0 a 100\% de parasitismo, enquanto que após a formação da cabeça foram encontrados de 2 a 99 ovos por seis plantas, com 0 a $83 \%$ de parasitismo. Entretanto, a resposta de $T$. pretiosum foi independente da densidade de ovos, pois a proporção de ovos parasitados em função da densidade de ovos foi constante ao aumentar a disponibilidade de ovos em ambas as idades fenológicas de plantas estudadas (Figs 4b,d). As proporções de ovos parasitados (média \pm EP) em plantas antes e após a formação da cabeça foram de 0,35 \pm 0,07 e 0,56 \pm 0,04, respectivamente. Esses índices de parasitismo diferiram entre as idades de plantas sendo superior em plantas após a formação da cabeça $\left(\mathrm{F}_{1,46}=8,20 ; \mathrm{P}=0,0063\right)$.

\section{Discussão}

O conhecimento do padrão de oviposição da praga alvo 

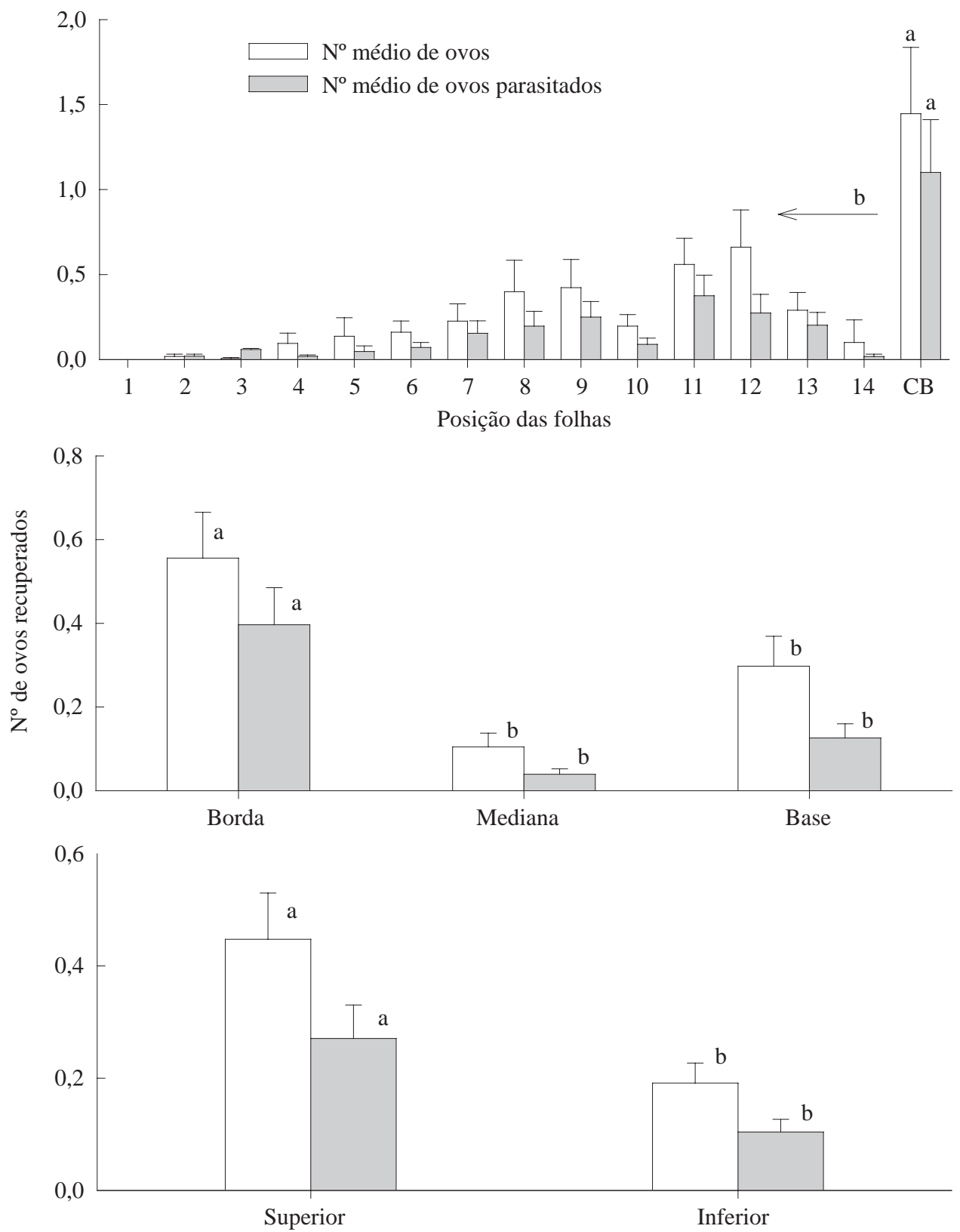

Fig 3 a) Número médio de ovos de Plutella xylostella e ovos parasitados por Trichogramma pretiosum por folha e cabeça (CB) de plantas de repolho, Brassica oleracea var. capitata (Midori) com 60 dias após o transplante; b) partes da folha; c) epiderme da folha. As médias (+EP) seguidas de mesma letra na categoria, não diferem entre si, pelo teste de Scott-Knott a 5\% de significância. Seta em a indica que todos os demais resultados das folhas diferiram da cabeça (CB).

é muito importante para levantamentos populacionais, bem como para a constatação do parasitismo natural e análise de eficiência de liberações de parasitóides. Neste estudo, ficou evidenciado que $P$. xylostella prefere ovipositar em folhas mais jovens de plantas de repolho. Os resultados da presente pesquisa corroboram os estudos de Badenes-Perez et al (2006), que constataram 3,7 vezes mais posturas de $P$. xylostella em folhas jovens do que em folhas mais velhas da planta. Esse comportamento pode ser repetido pela mariposa mesmo já havendo larvas colonizando essas folhas, o que leva à diminuição do risco de parasitismo das larvas, por exemplo (Shiojiri \& Takabayashi 2003). a
A variação no sítio de oviposição de $P$. xylostella em plantas de repolho de acordo com a idade da planta traz consequências para o seu controle. A alocação da descendência em maior profundidade na copa das plantas (parte basal das folhas), além de escapar relativamente do parasitismo por $T$. pretiosum, como verificado neste estudo, também fornece maior proteção a outros fatores de mortalidade às larvas oriundas desses ovos, especialmente, insolação e contato com inseticidas. Como a larva apresenta comportamento de minar as folhas no primeiro instar, o efeito de escape torna-se, ainda, mais pronunciado. Estudos suportam tais hipóteses, pois o tamanho e a complexidade 

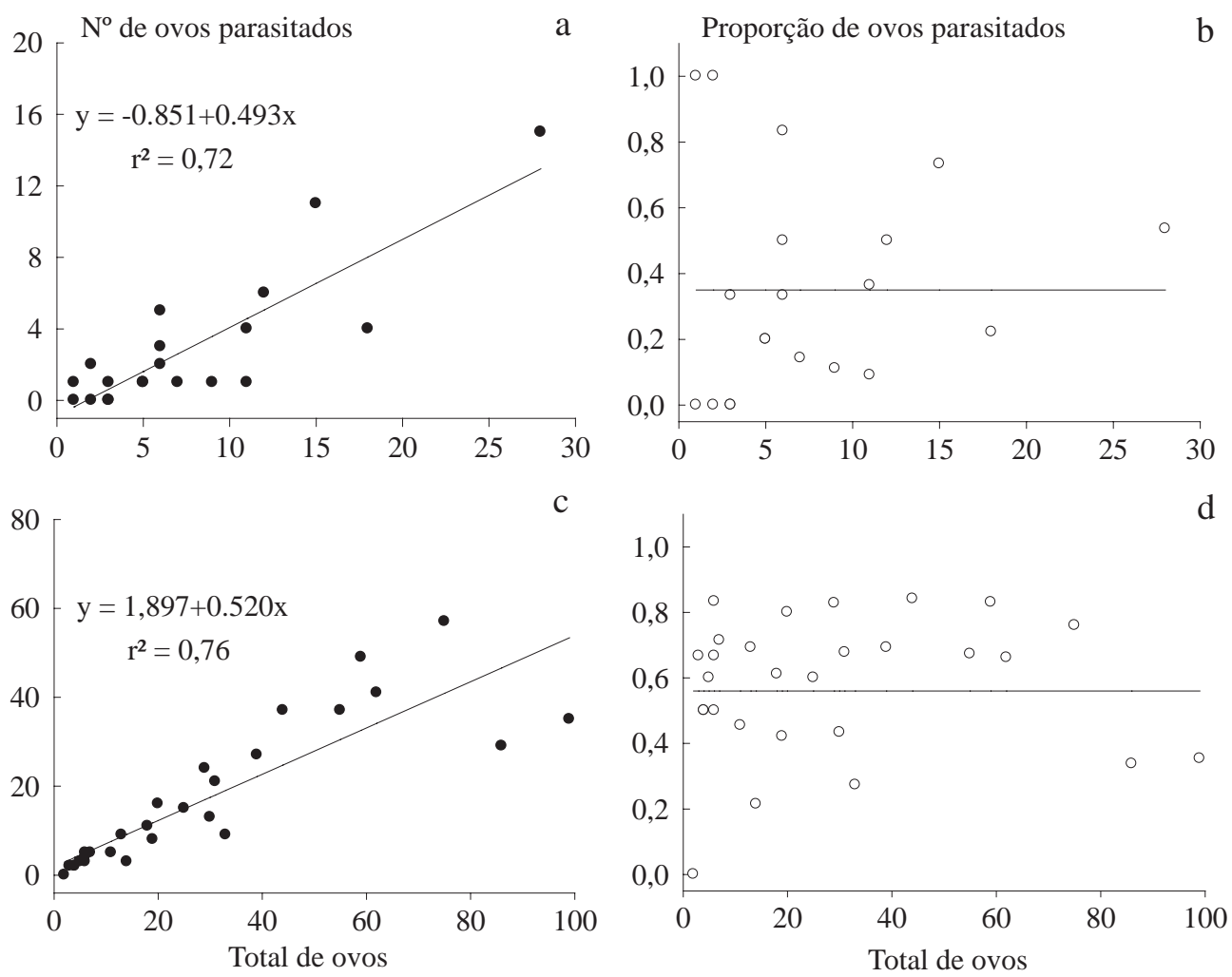

Fig 4 Número de ovos de Plutella xylostella parasitados por Trichogramma pretiosum em função do número de ovos recuperados por seis plantas (repetição) de repolho, Brassica oleracea var. capitata e proporção de ovos parasitados antes da formação da cabeça (a e b) e; após a formação da cabeça (c e d), respectivamente. Nota: valores dos eixos "x" e “y” diferem em função dos resultados; a linhas em b e d correspondem às médias das proporções de ovos parasitados.

da planta podem exercer influência em insetos-praga, bem como afetar o desempenho dos inimigos naturais, resultando em menores taxas de parasitismo (Gingras \& Boivin 2002, Gingras et al 2003, Reddy et al 2004, Hamilton et al 2005, Romeis et al 2005). Entretanto, em plantas após a formação da cabeça, $P$. xylostella é direcionada a ovipositar em locais mais expostos, de acesso mais fácil para as mariposas que as plantas de repolho antes da formação da cabeça, que são menores em área foliar e apresentam maior espaço entre as folhas. Assim, o acesso dos adultos à base das folhas é facilitado, o que acarretou maior oviposição nessa parte da folha, quando comparado com plantas após a formação da cabeça.

Com relação a plantas de repolho com cabeça formada, os adultos de P. xylostella concentraram sua oviposição em partes mais jovens das plantas, como as folhas mais próximas do centro e região da cabeça, como sugerido pelos resultados de Badenes-Perez et al (2006), e, consequentemente, mais expostas, resultando em maior taxa de parasitismo nessa idade da planta, bem como na folha e sua superfície superior (Figs 2a-c, 4a-d).

O número de ovos parasitados por $T$. pretiosum foi relacionado com a densidade de ovos, bem como com o local de oviposição de $P$. xylostella, sendo superior nos locais de maior número de ovos depositados pela praga como na folha da cabeça (Fig 2a). Em plantas antes da formação da cabeça onde houve distribuição homogênea dos ovos entre as folhas das plantas, também, não houve diferença no parasitismo. O comportamento de parasitismo associado a locais de maior densidade do hospedeiro indica que T. pretiosum é retido em locais de maior disponibilidade de hospedeiros (Pratissoli et al 2003). No entanto, apesar do maior número de ovos na base das folhas de repolho (Fig 2a), entre as regiões da folha (base, mediana e borda) antes da formação da cabeça, o parasitismo não se concentrou nesse local. Foi maior na superfície superior da folha, que é mais acessível e de maior luminosidade incidente, indicando que locais de maior profundidade na copa da planta permite, de certa forma, algum escape do parasitismo por T. pretiosum.

A taxa de parasitismo encontrada mostra que T. pretiosum respondeu independente da densidade de ovos do $P$. xylostella, pois mesmo com o aumento na disponibilidade de ovos no ambiente, a taxa de parasitismo foi constante (Figs 4a,c), caracterizando resposta funcional do tipo I (Holling 1959). Esse comportamento de parasitismo de ovos por Trichogramma, no campo, também tem sido encontrado em outras espécies de Trichogramma e agroecossistemas (Wang et al 1997, Suh et al 2000, Hoffman et al 2002, Faria et al 2008). Uma das razões para tal resposta é a complexidade do ambiente, oriunda das interações entre a planta hospedeira e o comportamento de oviposição da praga, como observado neste estudo, impedindo que o parasitóide passe a localizar mais ovos e, consequentemente, expressar toda a sua capacidade reprodutiva.

Com base nos resultados, podemos afirmar que houve efeito da estrutura das plantas de repolho nas idades antes 
e após a formação da cabeça e da quantidade de ovos no parasitismo por $T$. pretiosum. As altas densidades de ovos de P. xylostella em determinados locais da planta, como na cabeça, podem ter facilitado a sua localização por T. pretiosum, possivelmente devido a cairomônios do hospedeiro presente no local de oviposição (Vinson 1997, Wang et al 1997, Gingras \& Boivin 2002) e sua posterior retenção no local. Isso pode explicar o maior parasitismo (56\%) em plantas com idade após a formação da cabeça, pois também houve maior disponibilidade de ovos em comparação a plantas antes da formação da cabeça. Estruturas mais simples de plantas favorecem o parasitismo por T. pretiosum, como a região da borda e a epiderme superior da folha envolvendo a cabeça de repolho, que ficam mais expostos em comparação à base das que tiveram maior oviposição em plantas antes da formação da cabeça. Entre diferentes plantas de brássicas (repolho, brócolis e couve-de-Bruxelas), Gingras et al (2003) concluíram que o maior parasitismo de ovos de $P$. xylostella por T. pretiosum obtido em repolho ocorreu por ter essa planta menor complexidade de copa e arranjo das folhas que as de brócolis e couve-de-bruxelas.

Os índices de parasitismo observados, apesar de serem considerados relativamente altos para agentes de controle biológico (35\% e 56\%), não são suficientes para a supressão da praga em questão. É importante ressaltar que esses índices de parasitismo poderão ser aumentados após a seleção de linhagens adequadas ao agroecossistema em questão, às condições climáticas da região e adaptadas à praga-alvo. A determinação do número ideal de parasitóides a serem liberados por ovo hospedeiro, como preconizado em programas de controle biológico com Trichogramma também deve aumentar os índices de parasitismo (Parra \& Zucchi 2004). No entanto, o sucesso no controle biológico no sistema aqui estudado também dependerá da aquisição de outros conhecimentos que envolvam a relação planta - $P$. xylostella - T. pretiosum, assim como a capacidade de busca do parasitóide no ambiente a ser liberado.

Os resultados desta pesquisa indicam os locais na planta e parte da folha onde se deve buscar a estimativa do número de ovos de $P$. xylostella por planta e que resultados poderão ser esperados com liberações de $T$. pretiosum em plantas de repolho antes e após a formação da cabeça.

\section{Agradecimentos}

À Dra Ranyse Barbosa Querino da Silva (Embrapa Roraima) pela identificação de Trichogramma pretiosum e a Leandro Bacci (UFV) pelo auxílio na aplicação do teste de Scott-Knott. À Dra Ângela Maria Isidro de Farias e Dra Elizabeth Araújo de Albuquerque Maranhão pela contribuição nas melhorias do trabalho. À CAPES pela bolsa de estudos ao primeiro autor e ao Programa PROCAD CAPES no. 83054.

\section{Referências}

Altre J A, Vandenberg J D (2001) Factors influencing the infectivity of isolates of Paecilomyces fumosoroseus against diamondback moth, Plutella xylostella. J Invertebr Pathol 78: 31-36.

Badenes-Perez F R, Nault B A, Shelton A M (2006) Dynamics of diamondback moth oviposition in the presence of a highly preferred non-suitable host. Entomol Exp Appl 120: 23-31.

Barros R, Vendramim J D (1999) Efeito de cultivares de repolho, utilizados para criação de Plutella xylostella (Lepidoptera: Pluellidae), no desenvolvimento de Trichogramma pretiosum Riley (Hymenoptera: Trichogrammatidae). An Soc Entomol Brasil 28: 469-476.

Charleston D S, Rami K W, Dicke M, Vet L E M (2006) Impact of botanical extracts derived from Melia azedarach and Azadirachta indica on populations of Plutella xylostella and its natural enemies. Biol Control 39: 105-114.

Faria C A, Torres J B, Fernandes A M V, Farias A M I (2008) Parasitism of Tuta absoluta (Meyrick) in tomato plants by Trichogramma pretiosum Riley in response to host density and plant structures. Rev Ciên Rural 38: 1504-1509.

Gingras D, Boivin G (2002) Effect of plant structure, host density and foraging duration on host finding by Trichogramma evanescens (Hymenoptera: Trichogrammatidae). Environ Entomol 31: 1153-1157.

Gingras D, Dutilleul P, Boivin G (2003). Effect of plant structure on host finding capacity of lepidopterous pests of crucifers by two Trichogramma parasitoids. Biol Control 27: 25-31.

Godin C, Boivin G (1998) Lepidopterous pests of Brassica crops and their parasitoids in southwestern Quebec. Environ Entomol 27: 1157-1165.

Hamilton A J, Endersby N M, Ridland P M, Neal M (2005) Effects of cultivar on oviposition preference, larval feeding and development time of diamondback moth, Plutella xylostella (L.) (Lepidoptera: Plutellidae), on some Brassica oleracea vegetables in Victoria. Aust J Entomol 44: 284-287.

Holling C S (1959) Some characteristics of simple types of predation and parasitism. Can Entomol 91: 385-398.

Ibrahim A M A, Kim Y (2006) Parasitism by Cotesia plutellae alters the hemocyte population and immunological function of the diamondback moth, Plutella xylostella. J Insect Physiol 52: 943-950.

Kwon D H, Choi B R, Park H M, Lee S H, Miyata T, Clark J M, Lee S H (2004) Knockdown resistance allele frequency in field populations of Plutella xylostella in Korea. Pestic Biochem Physiol 80: 21-30.

Olson D M, Andow D A (2006). Walking pattern of Trichogramma nubilale Ertle \& Davis (Hymenoptera: Trichogrammatidae) on various surfaces. Biol Control 39: 329-335.

Parra J R P (1997). Técnicas de criação de Anagasta kuehniella, hospedeiro alternativo para produção de Trichogramma, p.121150. In Parra J R P, Zucchi R A (eds) Trichogramma e o controle biológico Aplicado. Piracicaba, FEALQ, 324p.

Parra J R P, Zucchi R A (2004) Trichogramma in Brazil: feasibility of use after twenty years of research. Neotrop Entomol 33: 271-281. 
Pratissoli D, Parra J R P, Fernandes O A, Oliveira R C, Zago H B, Pereira F F (2003) Patron de ovoposicion de la polilla del tomate, Tuta absoluta, en tomates bajo diferentes densidades de poblaciones adultas en invernadero. Rev Agroc Chile 19: 11-15.

Reddy G V P, Tabone E, Smith M T (2004) Mediation of host selection and oviposition behavior in the diamondback moth Plutella xylostella and its predator Chrysoperla carnea by chemical cues from cole crops. Biol Control 29: 270-277.

Romeis J, Babendreier D, Wäckers F L, Shanower T G (2005) Habitat and plant specificity of Trichogramma egg parasitoidsunderlying mechanisms and implications. Basic Appl Ecol 6: 215-236.

Sayyed A H, Attique M N R, Khaliq A (2005) Stability of fieldselected resistance to insecticides in Plutella xylostella (Lep., Plutellidae) from Pakistan. J Appl Entomol 129: 542-547.

Shelton A M, Nault B A (2004) Dead-end trap cropping: a technique to improve management of the diamondback moth, Plutella xylostella (Lepidoptera: Plutellidae). Crop Prot 23: 497-503.

Shiojiri K, Takabayashi J (2003) Effects of specialist parasitoids on oviposition preference of phytophagous insects: encounterdilution effects in a tritrophic interaction. Ecol Entomol 28: 573-578.

Suh C P C, Orr D B, van Duyn J W (2000) Trichogramma releases in North Carolina cotton: why releases fail to suppress Heliothine pests. J Econ Entomol 93: 1137-1145.

Torres J B, Freitas F S, Pratissoli D (1995) Avaliação de diferentes porcentagens da mistura de farinha de milho com farinha de trigo integral e levedura-de-cerveja na criação de Anagasta kueheniella (Zeller, 1879). Rev Ciên Prática 19: 365-368.

SAEG (1997) SAEG - Sistema de análises estatísticas e genéticas. Manual do usuário, versão 7.1. Viçosa, Universidade Federal de Viçosa, 150p.

Vinson S B (1997) Comportamento de seleção hospedeira de parasitóides de ovos, com ênfase na família Trichogrammatidae, p.67-119. In Parra J R P, Zucchi R A (eds) Trichogramma e o controle biológico aplicado. Piracicaba, FEALQ, 324p.

Wang B, Ferro D N, Hosmer D W (1997) Importance of plant size, distribution of egg masses, and weather conditions on egg parasitism of the European corn borer, Ostrinia nubilalis by Trichogramma ostriniae in sweet corn. Entomol Exp Appl 83: 337-345.

Zucchi R A, Monteiro R C (1997) O gênero Trichogramma na América do Sul, p.41-66. In Parra J R P, Botelho P S M, Ferreira B S C, Bento J M S (eds) Controle biológico no Brasil: parasitóides e predadores. São Paulo, Manole, 635p.

Received 24/VIII/08. Accepted 05/I/10. 